Archives

$24 \mid 2000$

La séduction

\title{
Le charisme en politique : idée séduisante ou concept pertinent?
}

Hinnerk Bruhns

\section{(2) OpenEdition}

Journals

\section{Édition électronique}

URL : http://journals.openedition.org/ccrh/1882

DOI : $10.4000 /$ ccrh. 1882

ISSN : $1760-7906$

Éditeur

Centre de recherches historiques - EHESS

Édition imprimée

Date de publication : 20 avril 2000

ISSN : 0990-9141

\section{Référence électronique}

Hinnerk Bruhns, «Le charisme en politique : idée séduisante ou concept pertinent ? », Les Cahiers du Centre de Recherches Historiques [En ligne], 24 | 2000, mis en ligne le 16 janvier 2009, consulté le 10 décembre 2020. URL : http://journals.openedition.org/ccrh/1882 ; DOI : https://doi.org/10.4000/ccrh 1882

Ce document a été généré automatiquement le 10 décembre 2020.

Article L.111-1 du Code de la propriété intellectuelle. 


\title{
Le charisme en politique : idée séduisante ou concept pertinent?
}

\author{
Hinnerk Bruhns
}

\section{À propos de la séduction du pouvoir}

1 Commençons par deux affirmations : le concept sociologique de charisme, utilisé pour l'analyse de formes de domination, n'a pas sa place dans une histoire de la séduction; la séduction n'est pas l'élément central d'une configuration charismatique. Contester d'emblée l'existence d'un lien utile entre le charisme en politique et une histoire de la séduction me semble une précaution nécessaire pour éviter toute confusion entre ces deux termes.

2 Car celui de séduction est souvent appliqué au champ politique. On parle ainsi couramment des masses séduites. Dans l'historiographie allemande, c'est l'esprit séduit de la bourgeoisie - plus exactement sa "séductibilité » (Verführbarkeit des Geistes) : manque de résistance des couches cultivées à des « séducteurs " politiques - qui depuis le livre de Helmuth Plessner, Die verspätete Nation ${ }^{1}$, est cité comme un des facteurs qui expliqueraient la faillite de la République de Weimar. Le terme de séduction peut donc glisser du privé et de l'individuel au public, au collectif et au politique. Dans le langage courant, un lien est facilement établi entre les qualités charismatiques d'un chef et le phénomène de séduction. Non seulement dans le langage de tous les jours, mais aussi dans des textes de sociologie, d'histoire et de psychologie. On parle ainsi du charisme d'un chef qui séduit. Dans La Psychologie des foules de Gustave Le Bon, il n'y a pas le mot de charisme. Mais la chose, telle qu'on l'imagine en général, y est :

[...] le type du héros cher aux foules aura toujours la structure d'un César. Son panache les séduit, son autorité leur impose et son sabre leur fait peur².

En politique, la séduction, comme technique, vise à la fois des individus, des groupes et la masse. Le charisme, entendu comme une force de séduction, est censé s'exercer sur la masse, la foule. Pour Gustave Le Bon, la foule était femme. Hitler avait lu et relu La Psychologie des foules. Serge Moscovici observe que Hitler 
[...] ne s'est pas contenté de penser qu'il faut traiter la foule comme une femme, il a aussi imaginé une stratégie pour traiter les femmes comme une foule³.

Et il cite une phrase du philosophe Ernst Bloch à propos de l'adhésion des femmes au nazisme :

Ici, c'est avec les séducteurs que ça a commencé. Des sentiments s'enflammèrent, des cœurs volèrent dans cette direction. Qu'il y ait eu dans le tas un nombre de cœurs de femmes n'étonnera personne: on sait que les sentiments, c'est leur fort. Mais les choses ne sont pas si simples, toutes les femmes ne sont pas faites que d'instincts, et ce n'est pas la brosse que Hitler porte sous le nez qui a suffi à les attirer.

4 En effet, les choses ne sont pas si simples, et la psychologie sociale a développé depuis des concepts et des modèles plus opératoires. Est-ce la mystique du pouvoir qui explique l'attirance qu'exerçait Hitler sur nombre de femmes, surtout jeunes, dont quelques-unes allèrent jusqu'à des tentatives de suicide 4 ? Dans ce contexte, la figure du "séducteur " est de peu d'utilité, me semble-t-il pour saisir la nature du phénomène. Certes, la montée du national-socialisme s'est appuyée sur de véritables opérations de séduction dont la plus efficace visait la jeunesse, en particulier les garçons. Le charisme du Führer y a joué un rôle important, mais non décisif. La technique était plus fine: offrir aux jeunes garçons une vie en groupe, une vie souvent d'aventures, sous le commandement d'un " chef », à peine plus âgé qu'eux.

5 Il arrive aussi que la séduction et le pouvoir de séduction soient décrits par des mots qui se rapprochent du phénomène de domination auquel est assimilé, depuis le début du siècle, dans les sciences sociales, le concept de charisme. Dans la citation qui suit, il s'agit évidemment du pouvoir des femmes (pouvoir dont elles ne seraient d'ailleurs pas conscientes). Pour Jean Baudrillart, « séduction et féminité se confondent » :

[...] la séduction représente la maîtrise de l'univers symbolique, alors que le pouvoir ne représente que la maîtrise de l'univers réel. La souveraineté de la séduction est sans commune mesure avec la détention du pouvoir politique ou sexuel.

Mais ce pouvoir s'exerce apparemment dans un monde artificiel et sans substance. Car, poursuit Baudrillart,

Le plus bel exemple en est sans doute dans la seule grande constellation de séduction qu'aient produite les temps modernes: celle des stars et des idoles de cinéma ${ }^{5}$.

6 Une telle vision du pouvoir de la séduction paraît pâle, comparée au pouvoir révolutionnaire du charisme tel que le définissent aujourd'hui les sciences sociales, pouvoir par lequel des forces irrationnelles ou archaïques font irruption dans notre monde moderne, rationnel et désenchanté. Certes, dans le langage courant, les termes se confondent. Des stars du cinéma ou du sport sont qualifiées de charismatiques, et ce mot prend parfois tout simplement la signification de grandeur, de qualité exceptionnelle, etc. Employé dans ce sens, le mot est sans intérêt pour l'historien qui est à la recherche de concepts opératoires pour rendre compte de situations historiques concrètes, c'est-à-dire dotés d'une véritable valeur heuristique. Les usages courants du terme "charisme» reflètent deux difficultés auxquelles l'historien est confronté :

- 1. Celle, générale, d'utiliser des concepts qui définissent ou décrivent non seulement des relations sociales mais des relations et effets psychologiques entre des acteurs, et, de plus, entre acteurs individuels et acteurs collectifs ;

- 2. Celle, ensuite, dans le cas des notions de séduction et de charisme, d'avoir affaire à deux concepts de statuts très différents : le premier est un terme du langage courant et partiellement du langage psychologique et de la psychanalyse ; un terme qui désigne aussi, 
dans le langage des sociologues et historiens, une pratique sociale ; du second, issu $\mathrm{du}$ domaine religieux, les sciences sociales ont fait une construction théorique, un instrument d'analyse sociologique, anthropologique et historique. Certes, ce concept fait également appel à des notions psychologiques cherchant à décrire une configuration et une structure sociale très complexes, qui, bien entendu, peuvent éventuellement englober des phénomènes ou des pratiques de séduction.

\section{À propos du concept de charisme}

7 Dans les sciences sociales, le concept de charisme ne peut plus être utilisé sans référence au sens que Max Weber lui a donné. Weber a emprunté le mot au vocabulaire théologique, l'a sécularisé et généralisé, et il en a fait un des concepts clefs de sa sociologie de la domination et de sa sociologie des religions. Les débats, dans les sciences sociales aujourd'hui, sur le concept de charisme ont tous, sans aucune exception, pour point de départ le concept wébérien. Il en va ainsi également des récentes tentatives, en anthropologie et en sociologie, de repenser et de redéfinir ce concept, notamment en mettant l'accent sur l'origine et la nature stigmatique de la qualité charismatique ${ }^{6}$. Depuis le début du siècle, l'intérêt pour la notion de charisme n'a d'ailleurs jamais disparu. En science politique, par exemple, elle a été longtemps utilisée pour analyser les formes d'autorité dans les pays en voie de développement.

Depuis une quinzaine d'années, les débats ont pris un nouvel essor, tant en sociologie qu'en anthropologie et même parmi des historiens. En France, en revanche, les sciences sociales donnent l'impression de ne pas trop s'y intéresser. Il arrive même qu'on rejette purement et simplement le concept de charisme, comme le fait Louis Dumont dans L'Idéologie allemande. Il y dit, à propos de l'incarnation du politique dans une personne :

Le cas qui nous intéresse ici est celui où un conflit insoluble mettant en jeu des systèmes d'idées, disons sinon des rationalités du moins des rationalisations opposées, oblige à recourir au règne d'un homme. Là où l'on voit le plus souvent une concentration du pouvoir, je voudrais attirer l'attention sur un autre aspect, celui pour lequel Max Weber a employé le mot, emprunté à la théologie et aujourd'hui très répandu, de charisme. Le mot n'éclaircit rien, il obscurcit plutôt ce dont il s'agit. L'intervention d'une personne humaine rouvre tout un monde de perceptions et de sentiments que le débat d'idées prétendait ignorer : il peut de nouveau y avoir respect, admiration, attachement, dévouement, identification de la part du sujet-citoyen, et même, osons le mot, soulagement d'une tension sentie à la fois comme extrême et comme étroite. Je ne fais ici que gloser pour la clarté ce qui, dans le langage de Talcott Parsons, s'appellerait une transition d'une orientation universaliste et spécifique - c'est-à-dire rationnelle - à une orientation particulière et diffuse, c'est-à-dire traditionnelle... ${ }^{\text {. }}$.

Dans L'Âge des foules, Serge Moscovici donne un jugement opposé :

Aujourd'hui le mot charisme est devenu si populaire que même les journaux à grand tirage l'utilisent, le supposant compris de leurs lecteurs. Sa fortune doit beaucoup à son obscurité et à son imprécision. Il éveille en nous des échos mystérieux. En revanche, les idées de Max Weber, son inventeur, sont beaucoup plus claires...

Et Moscovici résume ensuite admirablement certains aspects du charisme dans l'œuvre de Weber, surtout le charisme du prophète. Pourtant, dans cette septième partie de son livre, partie intitulée «La psychologie du chef charismatique», il se détourne assez rapidement de Weber et poursuit sa propre « préoccupation principale » qui est :

[...] expliquer ce qu'est l'élément charismatique. Pourquoi séduit-il les foules? 
Le chef charismatique se trouve réduit chez Moscovici au rôle du «meneur décrit par les psychologues des masses ». Il constate que :

[...] le pouvoir charismatique recouvre exactement cette réalité. Ce que nous avons dit du prestige, de son caractère personnel et symbolique, du magnétisme exercé sur les masses, de la foi spontanée, de l'obéissance sans contrainte, de l'admiration qu'elles vouent au meneur, tout cela s'applique aussi bien au charisme. Entre les deux notions il n'y a pas de différence essentielle sinon que le charisme a un côté plus prophétique et le prestige un côté plus affectif qui le met a l'origine de toute forme de pouvoir. La théorie du prestige a précédé, voire inspiré, la théorie du charisme. En tout cas, proposées à peu près à la même époque, elles ont tenté de résoudre le même problème politique : celui du gouvernement et de la démocratie dans une société de masse ${ }^{8}$.

C'est une interprétation pour le moins contestable. Certes, le concept de "meneur » et celui de " prestige » (« le plus puissant ressort de toute domination ») ${ }^{9}$ définis par Gustave Le Bon en 1895 préfigurent plusieurs éléments de la théorie wébérienne du charisme. Mais Le Bon ramène tout à des facultés extraordinaires (innées ou acquises) qui font que l'on

[...] obéit [aux meneurs] comme la bête féroce obéit aux dompteurs qu'elle pourrait si facilement dévorer.

Le concept de "prestige» est pensé indépendamment du contenu du message charismatique et de la situation sociale, politique ou économique. Ce que sera le problème central pour Max Weber, la confirmation (Bewährung) du charisme, sa transformation, sa "quotidianisation", son institutionnalisation éventuelle, est évacué par deux phrases plates:

L'homme qui réussit, l'idée qui s'impose, cesse par ce fait même d'être contesté. Le prestige disparaît toujours avec l'insuccès ${ }^{10}$.

En ce qui concerne Louis Dumont, je ne sais si par son rejet du concept de charisme, il exprime un sentiment largement partagé. Il est certain qu'une lecture de Weber aurait pu l'éclaircir plus que celle de Talcott Parsons ${ }^{11}$. Mais la traduction française de Économie et société n'est pas bonne et des passages importants concernant le charisme soit sont inexacts soit disent le contraire de ce que Weber a écrit. En plus, le grand chapitre sur Die charismatische Herrschaft und ihre Umbildung (La domination charismatique et sa transformation) ${ }^{12}$, qui fait partie de la sociologie de la domination dans Économie et société, n'a toujours pas été traduit en français.

11 Du moins Louis Dumont a-t-il soulevé une réelle question, celle de l'utilité pratique du concept de charisme. C'est la seule question à laquelle je veux tenter de répondre dans les pages qui suivent. Je procéderai de façon très pragmatique en résumant d'abord quelques aspects du concept de charisme. Ensuite je présenterai brièvement deux cas concrets, pour l'analyse desquels des historiens ou des sociologues ont utilisé la théorie wébérienne du charisma comme instrument d'analyse, et un troisième où, contre toute attente, cette analyse n'avait pas été faite jusque très récemment. Il serait facile de prendre des exemples dans une liste de personnages politiques très proches de la définition «idéaltypique » d'un chef charismatique, tels Napoléon, Lénine, Hitler, Gandhi, Mao Zedong, etc. Mais l'intérêt de concepts « idéaltypiques » réside justement dans leur utilité pour l'analyse de réalités historiques (ou sociologiques) qui se trouvent à des distances différentes de cette «construction de l'esprit » qu'est l'idéaltype. Je présenterai donc trois cas qui, du point de vue du concept du charisme, paraissent assez disparates et pour l'analyse desquels l'intérêt du concept de domination charismatique n'a pas la même valeur heuristique : Hitler, Bismarck et César. 


\section{Le concept de domination charismatique dans la sociologie weberienne}

12 La typologie de la domination qui comprend les trois «types purs» de la domination légitime (légale-rationnelle, traditionnelle et charismatique) est traitée à au moins quatre endroits dans l'œuvre de Weber. Dans l'ordre chronologique de leur rédaction :

13 1. Dans Wirtschaft und Gesellschaft, chapitre IX "Soziologie der Herrschaft ", rédigé entre 1910-11 et 1913-14. Ce long chapitre (plus de 300 pages) comporte huit sections dont seulement deux existent en traduction française ${ }^{13}$. L'importante section 5, "Die charismatische Herrschaft und ihre Umbildung ", n'existe pas en traduction française ;

14 2. Die drei reinen Typen der legitimen Herrschaft. Eine soziologische Studie, publication posthume (1922), reprise ensuite dans les Gesammelte Aufsätze zur Wissenschaftslehre sous le titre Die drei reinen Typen der legitimen Herrschaft. Ce texte n'a pas été inclus dans l'édition française (Essais sur la théorie de la science) ${ }^{14}$. La rédaction de ce texte est datée par certains de 1913, par d'autres de 1918-192015;

15 3. Dans l'introduction à la Wirtschaftsethik der Weltreligionen, rédigée en 1913 et publiée pour la première fois en 1915 dans l'Archiv für Sozialwissenschaft und Sozialpolitik (vol. 41). La typologie de la domination est traitée dans la deuxième partie de cette introduction, accessible maintenant en traduction française ${ }^{16}$. Le long passage sur les trois types est introduit ainsi: "Les sociétisations (Vergesellschaftungen) et les communautés ( Gemeinschaften) religieuses, quand elles sont pleinement développées, ressortissent du type des groupements de « domination » (Herrschaftsverbände)... »

16 4. Dans "Die Typen der Herrschaft », chapitre III de la première partie de Wirtschaft und Gesellschaft, rédigé dans les années 1918-192017.

17 Les différences entre ces quatre versions ne nous concernent pas ici. Voici simplement quelques aspects importants de la théorie wébérienne de la domination charismatique.

18 Le mot grec « charisme " (grâce, faveur) désignait un don religieux particulier dans les communautés du christianisme primitif. Il avait été employé d'abord par Rudolf Sohm (1841-1917), grand spécialiste de droit ecclésiastique et d'histoire du droit, pour caractériser la qualité religieuse exceptionnelle des prophètes de l'Ancien Testament et leur capacité extraordinaire d'influencer et de guider, de diriger les hommes. Weber définit le charisme comme une qualité, considérée comme "außeralltäglich » (extraquotidienne), d'une personnalité,

[...] en raison de laquelle elle est considérée comme dotée de forces et de qualités surnaturelles ou surhumaines, ou au moins spécifiquement extra-quotidiennes qui ne sont pas accessibles à tous, ou comme envoyée par Dieu, ou comme exemplaire, et qui pour cette raison est considérée (estimée) comme « chef $»^{18}$.

19 La domination charismatique consiste dans la soumission (abandon) (Hingabe) à une personne considérée comme qualifiée de cette sorte, et à l'ordre révélé ou créé par celleci (WuG, p. 124 ; É\&S, p. 289). Par l'élément personnel, elle se différencie de la domination légale-rationnelle; par son caractère extraquotidien, de la domination traditionnelle :

La création d'une domination charismatique dans le sens "pur", tel que nous l'avons décrit, est toujours le produit de situations extérieures inhabituelles, particulièrement de situations politiques ou économiques, ou de situations intérieures, psychiques, notamment religieuses, ou des deux ensemble; et elle a 
son origine dans une excitation qui est commune à un groupe d'hommes et dans

l'abandon à l'héroïque (das Heroentum) quelque soit son contenu ${ }^{19}$. certaines personnes qui semblent dotées de capacités exceptionnelles: prophètes, chamans, héros guerriers, organisateurs qui imposent (!) obéissance et reconnaissance. Les chefs charismatiques deviennent le centre d'une emotionale Vergemeinschaftung (WuG, p. 141 ; É\&S, p. 322), d'une "communautarisation émotionnelle " ${ }^{20}$. Par conséquent, la communauté regroupée autour du chef charismatique est un groupement de domination (Herrschaftsverband), auquel les individus appartiennent en vertu d'un lien purement affectif, et dont le fonctionnement est à l'opposé des normes et des règles de la vie quotidienne, notamment en ce qui concerne le système juridique et les règles de l'acquisition économique rationnelle. La domination charismatique est donc "spécifiquement irrationnelle» et "spécifiquement révolutionnaire»; elle est wirtschaftsfremd, étrangère à l'économie (WuG, p. 141-142; É\&S, p. 324). qui le fascine le plus est celle du prophète. Mais ce sont moins les mécanismes psychologiques de la relation entre le chef et la masse qui le préoccupent que les conditions structurelles de l'exercice de cette domination, de sa survie et de sa transformation. Car la domination charismatique « pure » est par nature instable. Weber lie toujours au charisme un autre concept, celui de la Veralltäglichung, la «quotidianisation» (et non la "routinisation", comme le disent certaines traductions françaises et italiennes à la suite de celle faite par Talcott Parsons) : dès que le charisme aspire à la durée, il se trouve exposé aux conditions du quotidien et aux pouvoirs qui dominent celui-ci, surtout aux intérêts économiques. (WuG, p. 661). Par conséquent, la théorie du charisme s'enrichit d'une réflexion sur les processus de transformation du charisme et sur les différentes formes de charisme qui peuvent en résulter (charisme héréditaire, charisme de fonction, charisme propre (Eigencharisma) qui peut être réinterprété dans un sens anti-autoritaire (herrschaftfremde Umdeutung des Charisma ${ }^{21}$ ), etc. On aboutit ainsi à une théorie des configurations politiques et sociales concrètes dans lesquelles des éléments charismatiques s'opposent à des éléments de domination traditionnelle ou légale-rationnelle, les détruisent ou au contraire participent à leur légitimation.

Je ne peux, ici, entrer dans les détails. Il faut souligner que la domination charismatique est certes caractérisée par la figure du Führer, chef et guide, et par la communautarisation émotionnelle. Mais dès qu'elle aspire à une durée elle produit des structures d'organisation - que Weber appelle charismatisch qualifizierte Verwaltungsstäbe -, des corps administratifs qualifiés de façon charismatique qui sont indispensables pour imposer la soumission. Le personnel du «corps administratif » est sélectionné en fonction de son charisme et de son dévouement personnel. Cette "administration » ne fonctionne pas selon des règles rationnelles mais de façon irrationnelle.

Pour l'historien, l'intérêt de cette part de la sociologie wébérienne est double :

1. En ce qui concerne l'analyse des relations entre le chef et un groupe ou la masse, la théorie du charisme chez Weber présente des avantages, si on la compare aux théories de Freud. D'après sigmund Freud, des formations de masses (Vergemeinschaftungen, communautarisations) reposent structurellement (triebstrukturell) sur les mêmes mécanismes que des interactions simples, telles l'hypnose ou l'état amoureux. Une masse se constitue quand de nombreux individus investissent de la libido narcissique dans un 
seul et même objet, qui se trouve ainsi agrandi et élevé22. Si Freud démontre les forces affectives qui tiennent ensemble une communauté, il a tendance, sous l'influence de Gustave Le Bon, à surestimer la régression des capacités cognitives et à considérer les individus qui forment une masse comme des êtres mus principalement par des instincts. Chez Weber, au contraire, le charisme authentique et la relation sociale qu'il gouverne, sont du domaine du cognitif et du symbolique. Il n'y a pas de charisme sans la différenciation (culturelle et par le langage) entre quotidien et extra-quotidien, entre naturel-humain et surnaturel-surhumain, ni sans la représentation d'une force ou d'une puissance extraordinaire ;

2. La théorie du charisme intègre cet aspect psychologique dans une analyse multidimensionnelle (politique, économique, sociale, religieuse, culturelle) et permet d'analyser une situation ou une structure socio-politique complexe.

Je vais essayer d'illustrer cela maintenant à l'aide de trois exemples tirés de l'historiographie récente ${ }^{23}$.

\section{Trois exemples historiques}

\section{Hitler}

Dès le début des années 1940, l'historien Franz Neumann et le sociologue Hans Gerth, émigrés d'Allemagne aux États-Unis, avaient analysé le parti et le régime nationalsocialiste à l'aide des catégories wébériennes comme un mouvement et une domination charismatique ${ }^{24}$. Talcott Parsons également, comme le montre la récente publication de ses travaux sur le national-socialisme, avait essayé, à l'époque, de comprendre la dynamique de la domination national-socialiste à l'aide des catégories wébériennes de domination charismatique et de processus de "quotidianisation». Oubliées pendant longtemps, ces approches n'ont été reprises et prolongées qu'à partir des années 1980 . D'abord par R. M. Lepsius, suivi par d'autres sociologues parmi lesquels on peut citer Maurizio Bach et Uta Gerhardt, et dernièrement par l'historien britannique Ian Kershaw ${ }^{25}$ . Je ne retiens que quelques points de ces différents travaux.

1. Les analyses portent, dans un premier temps, sur les conditions de l'établissement d'une domination à caractère charismatique, à partir d'abord d'une situation charismatique latente, où la culture politique et la conscience de l'incapacité des institutions face à la crise économique et politique renforçaient la tendance à accepter un chef charismatique. La situation charismatique devient manifeste ensuite, quand la crise se conjugue avec l'apparition d'un prétendant qui promet de libérer l'Allemagne et de détruire l'ordre ancien, prétendant porté par des succès répétés. On assiste à la naissance d'une communautarisation émotionnelle (emotionale Vergemeinschaftung) des Allemands (non-juifs, bien entendu). Est étudié ensuite l'établissement de l'autorité charismatique sur le parti, le mouvement, d'abord; sur l'État ensuite. De façon comparable d'ailleurs aux niveaux micro et macro : destruction des règles et procédures formelles, suspension de la constitution, établissement de l'autorité personnelle absolue, absence de toute instance ou procédure institutionnelle à l'intérieur du parti. Contrairement à l'appellation Duce qui était pour Mussolini un titre, l'appellation Führer signifiait une rupture révolutionnaire : la suppression pure et simple d'une direction structurée de la nation ; 
2. Ensuite, étude de l'acceptation de la domination charismatique, à différents niveaux : les partisans convaincus, la perception, chez d'autres, qu'il n'y avait pas d'alternative, l'importance de la dynamique du processus révolutionnaire qu'il fallait maintenir en mouvement afin d'éviter les dangers de la "quotidianisation » : le charisme demande à être confirmée toujours de nouveau (Bewährung). Normes et règles furent dissoutes; ce qui renforçait la capacité d'imposer l'autorité ;

30 3. Finalement: analyse du fonctionnement, au niveau de ce que Weber appelait le Verwaltungsstab, la direction administrative, confiée à des partisans personnels. Dans la gestion de l'État, structures charismatiques et bureaucratiques coexistaient et interagissaient, et la bureaucratie devenait un instrument technique. Bien que la SS ait été organisée comme une bureaucratie, elle était autonome par rapport à toute législation et juridiction.

31 Dans l'analyse de Parsons, la forme particulière de la domination charismatique du national-socialisme

[...] se banalisait par une spirale de violence qui s'exerçait de plus en plus fortement contre des membres [du «mouvement »] et des chefs à un niveau inférieur, afin d'arriver par la force à réaliser l'utopie qui allait de pair avec le charisme. Dans le sultanisme de satrapes qui régissait la politique et la société, la terreur, soumise au processus de la "quotidianisation », devenait de plus en plus le trait caractéristique du régime charismatique et prenait selon la thèse de Parsons, des traits quasireligieux ${ }^{26}$.

Weber considérait comme le problème principal de la «quotidianisation» du charisme dans les sociétés modernes le

[...] passage de la direction et des principes administratifs charismatiques à la direction et aux principes administratifs quotidiens (É\&S, I, 334), (« Übergang von den charismatischen Verwaltungsstäben und Verwaltungsprinzipien zu den alltäglichen » (1976, p. 147)),

et jugeait comme peu probable que les appareils et structures des bureaucraties étatiques ne finissent par s'imposer, face aux mouvements charismatiques. Ce qu'on observe tout au long de l'histoire (brève) du régime national-socialiste va dans la direction opposée : la mise en place de directions administratives particulières, dépendant directement du Führer (führerimmediate Sonderstäbe, Bach p. $131 \mathrm{ff}$ ), d'appareils exécutifs particuliers ( Sonderexekutivapparate) dont le fonctionnement était à l'opposé des procédures bureaucratiques (p.136) avait pour conséquence non une bureaucratisation des structures d'exécution dépendant directement du Führer (führerunmittelbare Vollzugsstrukturen) mais une "charismatisation » partielle des structures traditionnelles de l'administration (p. 140 sq.).

L'intérêt de ces analyses - résumées ici de façon très sommaire - est qu'elles contribuent à sortir l'historiographie du national-socialisme de l'impasse dans laquelle elle s'est trouvée pendant longtemps: à savoir la querelle entre fonctionnalistes et intentionnalistes, donc entre des interprétations qui accordaient très peu d'importance au rôle de Hitler et celles, au contraire, qui étaient " hitlérocentriques ». S'interroger sur la nature du pouvoir de Hitler, sur sa domination charismatique, permet de combiner l'approche biographique avec une approche d'histoire sociale, comme le montre sa récente biographie par Ian Kershaw. De telles analyses permettent enfin une explication plus différenciée que celles qui essayaient d'appréhender la nature de la domination national-socialiste au moyen des concepts de dictature, de totalitarisme, de fascisme ou 
de domination d'un parti (Parteienherrschaft). Car le concept de domination ou autorité charismatique, pour citer Ian Kershaw :

[...] permet d'expliquer pourquoi ce régime a pu conjuguer une base de pouvoir aussi instable et un dynamisme extraordinaire - dynamisme qui, en raison de son incompatibilité foncière avec toute forme de gouvernement légal et rationnel, ne pouvait que se révéler destructeur et même, en fin de compte, autodestructeur. Bien plus, le concept de "domination charismatique» est précieux pour comprendre les bases de la suprématie de Hitler au sein du mouvement nationalsocialiste, son autonomie croissante dans le jeu complexe des relations de pouvoir à l'intérieur de l'État nazi et l'effet corrosif de son autorité lorsque celle-ci vint se superposer à une forme de domination qui lui était antinomique - l'appareil d'État allemand avec ses structures bureaucratiques et légales ${ }^{27}$.

34 Allant encore plus loin et s'appuyant sur les analyses de Parsons, Uta Gerhardt perçoit dans la «quotidianisation du charisme » le lien entre le système et les acteurs, entre l'assassinat de masse comme politique de l'État et la violence au niveau local et individuel (p. 531 sq.)

\section{Bismarck}

Ce deuxième exemple paraitra moins évident. C'est d'abord la personnalité de l'historien qui a proposé d'analyser le régime bismarckien comme une forme de domination charismatique qui retient l'attention: il s'agit de Hans-Ulrich Wehler, dans sa monumentale Deutsche Gesellschaftsgeschichte. D'après lui, manquait d'abord à Bismarck une qualité importante : celle de l'orateur et du démagogue, si important pour les chefs politiques charismatiques dans la théorie de Weber. Mais il y avait la fascination exercée par sa personnalité, par son autorité et il y avait le culte de sa personne. Manquait ensuite ce qui était si important chez Hitler: la Gefolgschaft, les partisans personnels, le parti transformé en «mouvement». Certes, il s'était rassemblé autour de Bismarck une sorte de « communauté charismatique » de partisans passionnément convaincus, mais ce n'est pas elle qui formait la base de sa domination politique. Ce n'est qu'après sa démission que Bismarck devint l'objet d'un véritable culte comme l'homme d'État, le guerrier et le héros le plus parfait qu'honoraient des centaines de tours (Bismarcktürme) érigées dans tout le pays, surtout à l'initiative de corporations estudiantines.

L'intérêt de cet exemple est qu'on passe d'une personnalité et d'une situation relativement proche de l'idéaltype (Hitler) à une réalité plus commune, à une configuration beaucoup moins marquée par la prédominance d'un des trois types de la théorie wébérienne. Le système de domination de Bismarck, entre 1862 et 1890 comportait, en termes wébériens :

- -des éléments de domination traditionnelle-la monarchie, les anciennes élites aristocratiques qui conservaient leur position, la foi dans la «sainteté » des valeurs et des normes traditionnelles ;

- •et des éléments de domination rationnelle - la légitimation de l'exercice du pouvoir par des procédures institutionnalisées dans les parlements de Prusse et de l'Empire (Landtag und Reichstag) ; la bureaucratie ; la régulation des conflits par la loi, etc.

Pourtant, selon Hans-Ulrich Wehler, on ne peut correctement appréhender la réalité de Preußen-Deutschland, de l'Allemagne prussienne entre 1862 et 1890 si on l'analyse comme une fusion de domination traditionnelle et rationnelle, sans prendre en compte un noyau 
central et prédominant de domination charismatique ${ }^{28}$. Les éléments principaux en sont, d'après Wehler :

- - La crise fondamentale grâce à laquelle Bismarck est parvenu à sa fonction; sa qualité de Eigencharismatiker en raison de sa capacité à surmonter et à régler toutes les crises intérieures et extérieures (trois guerres gagnées; règlement du conflit constitutionnel; fondation de l'État national ; unité allemande). Ce qui provoquait et renforçait la fascination et finit par produire un mythe. La Kanzlerdiktatur était une position autoritaire et dominante qui reposait sur l'acceptation et la force de fait () de son charisme qui avait fait ses preuves de nombreuses fois ;

- La gestion de la politique par Bismarck entre 1875 et 1890, allant d'une crise à l'autre, souvent provoquée ou manipulée, est lue par Hans Ulrich Wehler comme la défense, par le chancelier, de son charisme face aux forces politiquement fatales de la Veralltäglichung ("quotidianisation »), défense qui échoue à la fin.

Comme dans l'exemple précédent, le recours au concept de charisme apporte une clarification par rapport à des interprétations traditionnelles. Le système bismarckien a longtemps été désigné comme Kanzlerdiktatur ou encore de système bonapartiste. Wehler pense être plus près de la réalité en l'interprétant comme une domination charismatique (avec les réserves indiquées) qui constituait le noyau de la constitution réelle à l'intérieur de la structure d'une monarchie constitutionnelle. Cette interprétation n'est pourtant pas vraiment convaincante et elle a été contestée aussitôt ${ }^{29}$.

Certes, il arrive à Weber d'utiliser le concept de domination charismatique pour la catégorisation de formes de domination qui ne sont pas structurées par les mécanismes «a-personnels » de l'élection ou de la tradition. Utilisé ainsi, le concept engloberait la plupart des dictatures du $\mathrm{xx}^{\mathrm{e}}$ siècle. En revanche, l'élément central du concept wébérien ne se retrouve point chez Bismarck : l'idée de la domination charismatique comme le fait d'un chef prophétique, provenant des zones marginales de la société et capable d'enthousiasmer d'abord des partisans personnels, ensuite les masses, et de leur imposer sa volonté.

\section{César}

Avec César, nous retrouvons le thème de la séduction. Quand ses soldats entrèrent dans les rues de Rome, lors du triomphe gaulois, ils chantaient ces vers :

Citadins, surveillez vos femmes : nous amenons un adultère chauve. Tu as forniqué en Gaule avec l'or emprunté à Rome ${ }^{30}$.

Suétone dresse la longue liste des conquêtes du séducteur chauve, que Curion (le père) avait apostrophé dans un discours : "l'homme de toutes les femmes et femme de tous les hommes $»^{31}$. L'« exceptionnel pouvoir de séduction de Jules César $»^{32}$ nous permettra-t-il enfin de relier entre eux séduction et charisme ? Tout récemment, un historien français s'est demandé : « Qu'est-ce qui en lui plaisait tant aux femmes? » Voici sa réponse :

En bonne santé et soignant minutieusement sa personne, il réservait les élans d'un corps bien fait, aux membres proportionnés (Suétone), aux femmes de ses amis ou de ses ennemis, belle façon de remporter une double victoire! Il n'est pas jusqu'à sa calvitie, découvrant un large front, [...] qui attira les regards, car César resplendissait d'intelligence et de détermination. Plus d'une succombèrent sans qu'il se laissât dominer par Vénus qu'il «banalisait » en passant des bras d'une amante dans ceux d'une autre. Mais ce pouvoir de séduction dépassait la seule sphère de la sensualité. $S^{\prime}$ il savait enchaîner les cœurs et les corps, il se préparait, grâce à son charisme, à dominer le monde et à y installer son regnum ${ }^{33}$. 
Voilà comment on passe allègrement de la séduction au charisme et au règne du chef charismatique.

L'histoire de la séduction césarienne ne s'arrête pas là. De grands esprits universitaires, surtout allemands, des XIX ${ }^{e}$ et $\mathrm{Xx}^{\mathrm{e}}$ siècles y ont succombé également. En 1924, Friedrich Gundolf, qui fréquentait le cercle de Stefan George, ne faisait que résumer un sentiment largement partagé quand il écrivait que parmi les merveilles du monde César était «l'homme le plus authentique » (der richtigste Mensch), que ni Périclès, ni Goethe, ni Michel-Ange n'étaient à sa hauteur :

Que nous adorions Dieu sous l'espèce de la Loi ou de la magie : il n'est pas de héros en qui la Loi ait pris forme si nette, la nature telle ampleur magique, que César. Aucun n'est à la fois si limpide, si fermé, si mystérieusement créateur ; si génial et si classiquement formé. Aucun n'est à ce point déterminé par le milieu et les circonstances, et en même temps à ce point reflet de l'ordre éternel. C'est pourquoi nous l'avons élu comme l'image la plus simple qui nous ait été donné du véritable chef $^{34}$.

41 Face à la "quintessence de toute grandeur humaine " (Inbegriff aller menschlichen Größe ) - (Goethe)-, à «l'individualité colossale »-(Hegel) $)^{35}-$, au «pilote indispensable »-(Napoléon III) ${ }^{36}$-, à «l'homme le plus magnifique»(der herrlichste Mensch) - (Nietzsche) - ou au «plus grand parmi les mortels »-(Burckhardt) -, il était logique que les historiens ne sentent pas le besoin d'analyser la domination exercée par César à l'aide de concepts sociologiques. Tout était contenu dans la personnalité et trouvait tout naturellement sa traduction institutionnelle dans le projet monarchique. Dans son grand livre Charisma. Geschichte des antiken Herscherkultes ${ }^{37}$, Fritz Taeger a étudié les honneurs que César a obtenus et le culte dont il a été l'objet.

Le charisme est ici un élément essentiel du culte des princes. Mais on ne trouve dans cet ouvrage aucune référence à la tradition sociologique du concept et au sens que Max Weber lui avait donné une génération auparavant. Plus près de nous, Zvi Yavetz a posé la question «César exerçait-il une domination charismatique?» (War Caesar ein charismatischer Herrscher ?). Mais ce n'était que pour la rejeter aussitôt :

Accoler à un Hitler ou à un César, à Churchill, Kennedy, Napoléon ou à Nasser

l'étiquette de "chef charismatique » ne nous aide pas vraiment à comprendre ces personnalités ${ }^{38}$.

Certes, mais il s'agit de comprendre leur pouvoir, non leur caractère.

La typologie des formes de domination légitimes est un instrument permettant d'analyser des configurations concrètes. L'objectif de l'analyse ne sera pas de classer des cas historiques en trois catégories sociologiques. Cela n'aboutirait qu'à des simplifications sans grand intérêt. L'objectif sera, dans un premier temps, de déterminer le degré de rapprochement du cas étudié avec un idéaltype, tel «la domination charismatique » ou "la domination traditionnelle», et de préciser le degré de présence d'éléments des différents types ou sous-types. Ce n'est donc pas un exercice sociologique gratuit, mais une tentative de précision et de différenciation. L'effort de conceptualisation n'est pas de nature théorique mais pratique, et c'est l'empirie, non la théorie, qui exige la construction d'idéaltypes.

Les sources antiques témoignent de la personnalité séduisante de César, et non seulement pour l'accuser d'adultère. Qu'il ait possédé du charisme est hors de doute : comme chef militaire, dans son manteau rouge de commandement, il était capable, dans des situations extrêmes, d'obtenir l'impossible de ses soldats ${ }^{39}$. De son vivant, déjà, il bénéficiait d'une 
sorte d'aura charismatique: c'est la légendaire "fortune" (tychè) de César. Mais ce charisme du chef militaire n'était pas le fondement de l'autorité de César sur son armée régulière, ni sur les troupes levées, juste avant et pendant la guerre civile. Les réformes introduites par Marius et l'évolution de la nature des guerres de conquête et des guerres civiles à la fin de la République avaient profondément changé la relation entre les soldats et les proconsuls qui obtenaient un commandement militaire extraordinaire. La personnalisation plus forte de cette relation pouvait, bien entendu, comporter des éléments charismatiques.

Est-ce qu'aux yeux de ses soldats César possédait un charisme au sens

[...] d'une qualité en raison de laquelle un personnage est considéré comme doté de forces et de qualités surnaturelles ou surhumaines, ou au moins spécifiquement extra-quotidiennes qui ne sont pas accessibles à tous, ou comme envoyé par Dieu, ou comme exemplaire, et qui pour cette raison est considéré comme "chef» [Führer $]^{40}$ ?

Malgré la transformation de l'armée romaine en une " institution d'aide aux sans-terre » (Versorgungsanstalt der Besitzlosen) ${ }^{41}$, il n'est pas possible de décrire l'armée de César comme une emotionale Vergemeinschaftung (communautarisation émotionnelle), ce qui est, selon Weber la qualité du groupement de domination, de la Gemeinde, dans la domination charismatique ${ }^{42}$. L'armée de César pouvait l'être dans des situations de détresse et de danger, mais elle ne l'était pas structurellement. En 49, quand César déclencha la guerre civile, il n'existait pas de situation charismatique dans laquelle les masses ou les soldats auraient réclamé un "chef», et César lui-même ne se réclamait aucunement d'une quelconque "mission» (Sendung). Il justifiait son action par ses propres «justes exigences ", ainsi que par celles de ses soldats, et par sa dignitas.

La domination absolue de César sur Rome, après sa victoire, reposait sur ses armées et sur les richesses des provinces, mais il ne gouvernait pas en tant que chef militaire et il n'a pu ni voulu régner, appuyé sur une seule Gefolgschaft, sur des partisans personnels dévoués. Il n'y avait non plus aucune composante charismatique dans la relation entre lui et ses partisans politiques, sénateurs ou chevaliers, qui l'avaient rejoint au début de la guerre civile. Son ascension politique, jusqu'au premier consulat, en 59 av. J.-C,. et pendant son proconsulat dans les Gaules, avait bénéficié de certains éléments traditionnels de la politique des populaires. Mais, à aucun moment de la guerre civile et de son bref règne, César n'a joué le rôle du chef populaire, comme le montrent la violence faite aux tribuns de la plèbe en 49 et les répressions sanglantes des révoltes de la plèbe en 48 et 47 , révoltes pourtant organisées et menées par des " partisans » de César. Ce n'est certainement pas un hasard si, pour toute la durée de la guerre civile et de la dictature de César, les sources antiques n'ont conservé aucun discours de César au peuple (contrairement à ceux prononcés devant ses soldats et devant le Sénat).

Déjà pendant la guerre, César avait instauré une structure administrative et politique parallèle, sorte de gouvernement de cabinet. Il serait erroné de l'assimiler à une direction administrative charismatique (charismatischer Verwaltungsstab). Car dans la domination charismatique,

[...] l'administration - dans la mesure où ce terme est adéquat - ne connaît aucune orientation selon des règles, qu'elles soient statuaires ou traditionnelles. Ce qui la caractérise, c'est la révélation et la création actuelle, l'acte et l'exemple, la décision de cas à cas. Par rapport à des ordres statuaires elle est irrationnelle ${ }^{43}$.

48 Le choix des personnes faisant partie du «cabinet» de César était fondé, outre sur la fidélité, sur la compétence et non sur la qualification charismatique. Loin de tendre à se 
substituer aux instances politiques traditionnelles, ces « fidèles » visaient et obtenaient ensuite, avec l'aide de César, les honneurs traditionnels, en particulier le consulat. En termes wébériens, cette partie de l'appareil de domination montrait eine rein patriarchale Struktur der Verwaltung, une structure purement patriarcale de l'administration. La description détaillée que Weber donne de ce type d'administration pourrait s'appliquer parfaitement au fonctionnement de la domus des empereurs romains qui était préfigurée dans une partie, mais une partie seulement, de l'administration de César dans les années 45 et 44 . Car ce n'est que là que

[...] la domination fut traitée comme un droit de propriété normal du seigneur ${ }^{44}$.

Ce type d'administration fait partie de la domination traditionnelle, sans qu'on puisse, bien entendu, caractériser la domination de César comme entièrement traditionnelle ou patriarcale.

Une analyse détaillée des élections et des nominations de magistrats depuis le début de la guerre civile jusqu'aux Ides de mars montre que le système politique traditionnel est absolument privilégié par rapport à ce qui pourrait paraitre le début d'une gestion privée de l'État. Si l'on y ajoute les élections et nominations réalisées ou prévues pour la durée de l'absence de César, qui devait partir pour trois ans en guerre contre les Parthes, on perçoit clairement que ces embryons de gestion privée ou parallèle, que César n'avait pas inventés mais élargis et perfectionnés, ne devenaient en aucune façon le support d'une transformation charismatique du système de gouvernement.

Les historiens ont souvent tenté de comprendre le règne de César comme l'institutionnalisation d'une monarchie fondée sur sa personnalité, si extraordinaire, sur ses visions et ambitions, sur un dessein de surmonter la crise. On oublie que l'élimination physique d'une grande partie de l'oligarchie sénatoriale n'avait été ni planifiée ni envisagée à l'avance. La position de domination absolue dans laquelle César s'est trouvé après les victoires de Thapsos (46 av. J.-C.) et de Munda (en 45) était inédite dans l'histoire de la République. Elle était le résultat d'une guerre civile dont trois continents avaient été le théâtre, mais qui ne s'était pas déroulée en Italie même.

51 La domination de César n'était pas de nature charismatique, mais elle finit par produire un charisme spécifique. Victoires militaires, accumulation d'honneurs excessifs, réformes gigantesques, activité débordante, largesses distribuées au peuple, avant et surtout après sa mort (par testament), mort tragique et exploitation habile par Antoine de cet assassinat, apparition opportune d'une étoile, création de légendes, troubles civils et militaires : nous voilà en présence d'un processus de construction d'une personnalité et d'une situation charismatiques qui se situait à la fin et non au début de la dictature de César - ce qu'Octavian a si bien su exploiter comme charisme héréditaire. Ce nouveau « charisme » aurait-il pu être pour César lui-même le fondement d'une légitimité durable et survivre à l'épreuve de la " quotidianisation »? On peut en douter.

Qu'apporte une telle lecture à la compréhension de la crise de la République romaine et à celle du règne de César? L'utilisation du concept de domination charismatique comme instrument d'analyse, même si celle-ci débouche sur un constat négatif, renforce l'interprétation de la crise de la fin de la République romaine, comme d'une « crise sans alternative $»^{45}$, ce qui nous amène à souligner plus fortement les éléments traditionnels et légaux-rationnels du règne de César. Sa dictature était l'expression d'une crise à laquelle il opposait sa virtuosité politique et militaire, son pouvoir et une multitude de réformes particulières. Bien que transgressant de plus en plus les normes de la société aristocratique, César ne se situait pas à l'extérieur de celle-ci. Il cherchait à être reconnu 
par ses pairs, par le Sénat et la noblesse, qui garantissaient la légitimité de l'ordre politique. Que César lui-même, en intervenant dans les institutions et en transgressant les règles, ait vidé l'ordre établi de son sens ne signifiait pas que le charisme individuel fût transféré sur une institution sociale, établi en règles et pérennisé. Au moment de la mort du dictateur, une solution structurelle de la crise n'était pas encore en vue. Une véritable domination charismatique au contraire, établie par l'irruption d'un mouvement ou d'une personnalité charismatiques dans l'histoire, aurait signifié une alternative, temporaire mais claire, à l'ordre traditionnel ${ }^{46}$.

\section{NOTES}

1. Helmuth Plessner, Die verspätete Nation. Über die politische Verführbarkeit bürgerlichen Geistes. Frankfurt am Main, Suhrkamp, 1974 ; la $1^{\text {re }}$ édition, rédigée aux Pays-Bas, avait paru à Zurich en 1935 sous le titre Das Schicksal des deutschen Geistes im Ausgang seiner bürgerlichen Epoche; le livre fut réédité sous le nouveau titre Die verspätete Nation, avec une nouvelle introduction, en 1959.

2. Gustave Le Bon, La Psychologie des foules (1 ${ }^{\text {re }}$ ed. 1895), Paris, 1963, p. 28.

3. Serge Moscovici, L'Âge des foules. Un traité historique de psychologie des masses (1 ${ }^{\text {re }}$ éd. 1981), nouvelle éd. entièrement refondue, Bruxelles, Éditions Complexe, 1991, p. 153.

4. Ian Kershaw, Hitler, 1889-1936, Hubris, trad. de l'anglais par Pierre-Emmanuel Dauzat, Paris, Flammarion, 1999, (page 31) ; édition originale : Ian Kershaw, Hitler, 1889-1936, Hubris, London, Allan Lane / The Penguin Press, 1998 ; Ian Kershaw, Hitler. Essai sur le charisme en politique, trad. de l'anglais par Jacqueline Carnaud et Pierre-Emmanuel Dauzat, Paris, Gallimard, 1995.

5. Jean Baudrillart, De la séduction, Paris, Aldine de Gruyter, 1979, p. 10, p. 19, p. 130.

6. Wolfgang Lipp, "Stigma und Charisma. Über soziales Grenzverhalten", in Zeitschrift für Kultursoziologie 1, 1985 ; Id., «Männerbünde, Frauen und Charisma. Geschlechterdrama im Kulturprozeß », in G. Vögler et K. von Welck, Männerbünde, Männerbande. Zur Rolle des Mannes im Kulturvergleich, Köln, 1990, vol. I, p. 31-40.

7. Louis Dumont, Homo aequalis II. L'Idéologie allemande. France-Allemagne et retour, Paris, Gallimard, 1991, p. 257.

8. Serge Moscovici, op. cit., p. 382, p. 387.

9. Gustave Le Bon, op. cit.

10. Ibid., p. 78, p. 82.

11. Un nouvel éclairage sur Parsons est pourtant apporté par Uta Gerhardt (éd.), Talcott Parsons on National Socialism. New York, 1993; Id., "Charismatische Herrschaft und Massenmord im Nationalsozialismus. Eine soziologische These zum Thema der freiwilligen Verbrechen an Juden », in Geschichte und Gesellschaft, 24, 1998, p. 503-538.

12. Max Weber, Wirtschaft und Gesellschaft. Grundriss der verstehenden Soziologie. $5^{\mathrm{e}}$ édition réalisée par J. Winckelmann (Studienausgabe), Tübingen, J. C. B. Mohr (Paul Siebeck), p. 654-687, traduction française: Max Weber, Économie et société, traduit de l'allemand par Julien Freund, Pierre Kamnitzer, Pierre Bertrand, Éric de Dampierre, Jean Maillard et Jacques Chavy sous la direction de Jacques Chavy et d'Éric de Dampierre, t. I, Paris, Plon, 1971, réimpr. en 2 vol. dans la collection « Agora Pocket », Paris, Plon, 1995.

Par la suite, l'édition allemande est citée sous la forme abrégée de WuG et l'édition française de 1995 sous la forme de É\&S. 
13. Section $n^{\circ} 6$ "Politische und hierokratische Herrschaft», dans Max Weber, Sociologie des religions, textes réunis et traduits par Jean-Pierre Grossein, introduction de Jean-Claude Passeron, Paris, Gallimard, 1996 ; section $n^{\circ} 7$ « Die nichtlegitime Herrschaft (Typologie der Städte) »; Id., La Ville, traduit par Philippe Fritsch, préface de Julien Freund, Paris, Aubier Montaigne, «Collection Champ urbain», 1982: ce dernier texte, publié de façon posthume dans Archiv für Sozialwissenschaft und Sozialpolitik en 1921, ne figurait pas dans le plan originel de Économie et Société.

14. Max Weber, Gesammelte Aufsätze zur Wissenschaftslehre, éd. par J. Winckelmann, Tübingen, J. C. B. Mohr (Paul Siebeck), $7^{e}$ éd., 1988 ( $1^{\text {ère }}$ éd. 1922), première publication dans Preußische Jahrbücher, vol.187, 1922, p. 1-12; dans la $4^{\mathrm{e}}$ édition de Wirtschaft und Gesellschaft, ce texte avait été intercalé, sans raison évidente, dans le chapitre " Soziologie der Herrschaft ».

15. Stefan Breuer, Max Webers Herrschaftssoziologie, Frankfurt/New York, Campus Verlag, 1991, p. 13 et p. 89 sq.

16. Max Weber, Sociologie des religions, p. 331-378 ; pour les formes de domination, p. 368 sqq. ; la première traduction française de l'«Introduction", Archives de sociologie des religions, 5, 1960, p. 5-30 ne comprenait pas cette partie du texte original.

17. Traduction française dans É\&S

18. WuG, p. 140, traduction $\mathrm{HB}$ (um derentwillen sie als mit übernatürlichen oder übermenschlichen oder mindestens spezifisch außeralltäglichen, nicht jedem andern zugänglichen Kräften oder Eigenschaften [begabt] oder als gottgesandt oder als vorbildlich und deshalb als "Führer" gewertet wird).

19. WuG, p. 661, trad. HB (Die Schöpfung einer charismatischen Herrschaft in dem geschilderten "reinen" Sinn ist stets das Kind ungewöhnlicher äußerer, speziell politischer oder ökonomischer, oder innerer seelischer, namentlich religiöser Situationen, oder beider zusammen, und entsteht aus der, einer Menschengruppe gemeinsamen, aus dem Außerordentlichen geborenen Erregung und aus der Hingabe an das Heroentum gleichviel welchen Inhalts).

20. Terme qui en général est malencontreusement traduit par «communauté émotionnelle ", p. 322.

21. $W u G$, p. 155 sqq., I, 350 sqq.

22. Stefan Breuer, op cit., p. 36 sq., à propos de Weber, Freud et Piaget.

23. On trouvera d'autres exemples dans quinze études de cas, allant de Périclès à Mao qui viennent d'être publiées par Wilfried Nippel (éd.), Virtuosen der Macht. Herrschaft und Charisma von Perikles bis Mao, Munich, C.H. Beck, 2000 ; Voir également son essai introductif «Charisma und Herrschaft », p. 7-22 ; et p. 281-289.

24. Franz Neumann, Behemoth. The Structure and Practice of National Socialism, 1933-1944, $2^{\mathrm{e}}$ éd., 1944 with new appendix, ( $1^{\text {re }}$ éd, 1942, réimpr., Londres, 1967), traduction allemande, Behemoth. Struktur und Praxis des Nationalsozialismus 1933-1940, Köln/Frankfurt am Main, 1977 ; Hans Gerth, "The Nazi Party: Its Leadership and Composition », American Journal of Sociology, 44, 1940, p.517-541; Arnold Zingerle, Max Webers historische Soziologie. Aspekte und Materialien zur Wirkungsgeschichte, Darmstadt, Wissenschaftliche Buchgesellschaft, 1981, p. 138 ; Uta Gerhardt (éd.), op. cit.

25. M. R. Lepsius, «Carismatic Leadership: Max Weber's Model and its Applicability to the Rule of Hitler ", in C. F. Graumann et S. Moscovici (éd.), Changing Conceptions of Leadership, New York / Berlin / Heidelberg / Tokyo, Springer Verlag, 1986, p. 53-66 (une version élargie de cet article a été publiée en allemand, «Das Modell der charismatischen Herrschaft und seine Anwendbarkeit auf den «Führerstaat » Adolf Hitlers », in M. R. Lepsius, Demokratie in Deutschland, Göttingen, 1993 ; Ian Kershaw, op. cit. ; Maurizio Bach, « Charismatische Herrschaft und die bürokratischen Mächte des Alltags in Hitlers Führerdiktatur ", in Arnold Zingerle (éd.), Carisma. Dinamiche dell'origine e della quotidianizzazione. Charisma. Dynamiken des Ursprungs und der Veralläglichung. = Annali di Sociologia-Soziologisches Jahrbuch, 9, 1993 - II, p. 127-144; et en version italienne «Dominio carismatico e quotidianità burocratica nella dittatura hitleriana", 
p. 145-164 ; Uta Gerhardt, «Charismatische Herrschaft und Massenmord im Nationalsozialismus. Eine soziologische These zum Thema der freiwilligen Verbrechen an Juden », Geschichte und Gesellschaft, 24, 1998, p. 503-538.

26. Uta Gerhardt, "Charismatische Herrschaft und Massenmord im Nationalsozialismus ", p. 517 : Diese Form der charismatischen Herrschaft veralltäglichte sich durch unentwegte Steigerung der Gewalt, die gegen Angehörige und untere Führer gerichtet wurde, um die allfällige Verwirklichung der Utopie, die mit dem Charisma vergesellschaftet war, zu erzwingen. Der Terror, der mehr und mehr zum Charakteristikum des Charisma-Regimes im Zuge der Veralltäglichung wurde, so die These, erhielt religionsähnliche Züge im politisch-gesellschaftlichen Satrapen-Sultanismus ; voir aussi p. 531.

27. Ian Kershaw, op. cit., p. 13 ; Ludolf Herbst, «Der Fall Hitler-Inszenierungskunst und Charisma ", in Wilfried Nippel (éd.), op. cit., p. 171-191 et p. 307-310.

28. Hans-Ulrich Wehler, Deutsche Gesellschaftsgeschichte, vol. III, Von der «Deutschen Doppelrevolution» bis zum Beginn des Ersten Weltkrieges 1849-1914, Munich, C. H. Beck Verlag. 1995.

29. John Breuilly, "Auf dem Weg zur deutschen Gesellschaft? Der dritte Band von Wehlers "Gesellschaftsgeschichte" ", Geschichte und Gesellschaft, 24, 1, 1998, p. 136-168, en particulier : p. 154 sqq.

30. Urbani, seruate uxores: moechum caluom adducimus. aurum in Gallia effutuisti, hic sumpsisti mutuum, Suétone, Divus Iulius, 51.

31. «Omnium mulierum uirum et omnium uirorum mulierem », Suétone, Divus Iulius, 52.

32. Robert Étienne, Jules César, Paris, Fayard, 1997, p. 66.

33. Robert Étienne, op. cit., p. 66 sq.

34. Friedrich Gundolf, César. Histoire et légende, traduit de l'allemand par Marcel Beaufils, Paris, Les Éditions Rieder, 1933, p. 8 ; édition allemande : Caesar. Geschichte seines Ruhms, Berlin, Georg Bondi, 1924, p. 8 : Ob wir Gott lieber als Gesetz oder als Zauber verehren : in keinem Heros wird das Gesetz so feste Gestalt, die Natur so reicher Zauber wie in Cäsar. Keiner ist so klar und dicht bei schöpferischem Geheimnis, keiner so sehr Genius bei klassischer Zucht, keiner so ganz bedingt durch Stätte und Stunde und doch gültiges Muster der wandellosen Ordnung. Darum wählen wir ihn als das schlichteste Bild des wahren Gebieters.

35. Friedrich Gundolf, Caesar im neunzehnten Jahrhundert, Berlin, Georg Bondi, 1926, p.46, traduction française in César. Histoire et légende, 1933, chap. IV.

36. Napoléon III, Histoire de Jules César, Paris, 1865-66, vol. II, p. 516.

37. Fritz Taeger, Charisma. Studien zur Geschichte des antiken Herrscherkultes, 2 vol., Stuttgart, Kohlhammer, 1957 et 1960.

38. Zvi Yavetz, Caesar in der öffentlichen Meinung, Düsseldorf, Droste Verlag, 1979, p. 210 sq.

39. Suétone, Divus Iulius, 65-70; en particulier 68 : quibus rebus et devotissimos sibi et fortissimos reddidit.

40. C'est la définition que donne Max Weber (WuG, p. 140, traduction HB) du charisme.

41. Max Weber, Économie et société dans l'Antiquité. Précédé de Les causes sociales du déclin de la civilisation antique, introduction de Hinnerk Bruhns, Paris, Éditions La Découverte, 1999, p. 351.

42. WuG, p. 141.

43. Der Verwaltung - soweit dieser Name adäquat ist - fehlt jede Orientierung an Regeln, sei es gesatzten, sei es traditionalen. Aktuelle Offenbarung oder aktuelle Schöpfung, Tat und Beispiel, Entscheidung von Fall zu Fall, jedenfalls also - am Ma(stab gesatzter Ordnungen gemessen - irrational, charakterisiert sie ; Max Weber, «Die drei reinen Typen der legitimen Herrschaft», in Gesammelte Aufsätze zur Wissenschaftslehre, p. 482.

44. Voir chez Weber, Wissenschaftslehre, p.479, la définition de la structure "purement patriarcale » de l'administration dont le type le «plus pur » est la domination sultanique.

45. Pour la notion de " crise sans alternative ", voir Christian Meier, Res publica amissa. Eine Studie zu Verfassung und Geschichte der späten Republik, Wiesbaden, Franz Steiner Verlag, 1966 ( $2^{\mathrm{e}}$ éd. 
1980) ; Christian Meier, Caesar, Berlin, Severin und Siedler, 1982, traduction française par Joseph Feisthauer, César, Paris, Seuil, 1989.

46. Pour plus de détails : Hinnerk Bruhns, « Caesar, der wahre Gebieter », in Wilfried Nippel (éd.), op. cit., p. 55-71 et p. 292-295. 